\title{
The Milagro anticenter hot spots: cosmic rays from the Geminga supernova? (Research Note)
}

\author{
M. Salvati ${ }^{1}$ and B. Sacco ${ }^{2}$ \\ 1 INAF - Osservatorio Astrofisico di Arcetri Largo Enrico Fermi 5, 50125 Firenze, Italy \\ e-mail: salvati@arcetri.astro.it \\ 2 INAF - Istituto di Astrofisica Spaziale e Fisica Cosmica via Ugo La Malfa 153, 90146 Palermo, Italy \\ e-mail: bruno.sacco@iasf-palermo.inaf.it
}

Received 15 February 2008 / Accepted 10 April 2008

ABSTRACT

Context. The Milagro experiment has announced the discovery of an excess flux of $\mathrm{TeV}$ cosmic rays from the general direction of the heliotail, also close to the Galactic anticenter.

Aims. We investigate the hypothesis that the excess cosmic rays were produced in the SN explosion that gave birth to the Geminga pulsar.

Methods. The assumptions underlying our proposed scenario are that the Geminga supernova occurred about $3.4 \times 10^{5}$ years ago (as indicated by the spin down timescale), that a burst of cosmic rays was injected with total energy $\sim 10^{49} \mathrm{erg}$ (i.e., about $1 \%$ of a typical SN output), and that the Geminga pulsar was born with a positive radial velocity of $100-200 \mathrm{~km} \mathrm{~s}^{-1}$.

Results. We find that our hypothesis is consistent with the available information. In a first variant (likely oversimplified), the cosmic rays have diffused according to the Bohm prescription (i.e., with a diffusion coefficient on the order of $c \times r_{\mathrm{L}}$, with $c$ the speed of light and $r_{\mathrm{L}}$ the Larmor radius). An alternative scheme assumes that diffusion only occurred initially, and the final propagation to the Sun was a free streaming in a diverging magnetic field.

Conclusions. If the observed cosmic ray excess does indeed arise from the Geminga SN explosion, the long-sought "smoking gun" connecting cosmic rays with supernovae would finally be at hand. It could be said that, while looking for the "smoking gun", we were hit by the bullets themselves.

Key words. ISM: cosmic rays - stars: supernovae: general - stars: supernovae: individual: Geminga

\section{Introduction}

In a recent paper (Abdo et al. 2008), the Milagro collaboration reports the detection of an excess of cosmic rays from the general direction of the heliotail, also close to the Galactic anticenter. The excess is diffuse but confined (in the following we assume a solid angle of 0.3 steradians to account for both hot spots A and $\mathrm{B}$ ), is composed of hadrons (photons and electrons are excluded to a highly significant level) and has a harder spectrum than the general cosmic ray background up to about $10 \mathrm{TeV}$.

The authors discuss some possible explanations and conclude that none of them is viable. In particular, because of the positional coincidence of the excess with the heliotail, they consider a local origin of the phenomenon, but discard it on the ground that several-TeV particles could not be easily generated or confined by the heliosphere.

In this Note, we revisit the hypothesis of a heliospheric origin of the hot spots and provide an additional quantitative argument against it. Then we point out that the closest plausible extra solar source is the supernova that produced the Geminga pulsar, and show that there is a region in the parameter space where this alternative hypothesis is valid.

\section{The heliospheric scenario}

All known effects of the heliosphere on the cosmic rays (solar modulation, anomalous cosmic rays) are detected at energies of about $1 \mathrm{GeV}$ per nucleon (e.g., Ngobeni \& Potgieter 2008), much lower than the several $\mathrm{TeV}$ observed by Milagro. On top of this, one can give a direct counter argument based on the energy budget. In the following, we take data about the cosmic rays from Longair (1981) and data about the Very Local InterStellar Medium (VLISM) from Axford \& Suess (1994).

From the Milagro paper (their Fig. 4) one deduces that the excess flux at $10 \mathrm{TeV}$ - measured as a fraction of the background cosmic ray flux - amounts to $15 \times 10^{-4}$ in region A and $6 \times 10^{-4}$ in region $\mathrm{B}$. These values refer to the cores of the two regions, which have a relatively small angular extent (for instance, the core of region $\mathrm{A}$ is only 0.02 steradians). To account for the lower level excess that is visible around the cores, we add two times the core counts in a solid angle of 0.3 steradians. Under these assumptions the average fractional excess is $5 \times 10^{-4}$, and the excess flux turns out to be

$\Phi \sim 5 \times 10^{-4} \times 0.3 \times 6.7 \times 10^{-6} \sim 1.0 \times 10^{-9} \mathrm{erg} \mathrm{cm}^{-2} \mathrm{~s}^{-1}$.

The acceleration region must be at least as large as one gyration radius, which for a $10 \mathrm{TeV}$ proton in a $1 \mu \mathrm{G}$ magnetic field is $r_{\mathrm{L}}=0.01$ parsec. Given the angular extent of the excess, the distance to the acceleration region must also be at least as much as $r_{\mathrm{L}}$, i.e., at least 20 times the distance to the heliopause. In the heliospheric hypothesis, the acceleration region is powered by the converging flows of the solar wake, and, at the inferred distance, the convergence angle must be $\sim 1 / 20$ radians. Finally, the 
power dissipated in the acceleration region (for a local velocity $v$ of $25 \mathrm{~km} \mathrm{~s}^{-1}$ and a local density $n$ of $0.1 \mathrm{~cm}^{-3}$ ), is approximately

$P \sim r_{\mathrm{L}}^{2} n m_{\mathrm{H}}\left(\frac{v}{20}\right)^{3} \sim 3.1 \times 10^{23} \mathrm{erg} \mathrm{s}^{-1}$.

This corresponds to a maximum flux at Earth equal to

$\Phi \sim \frac{P}{4 \pi r_{\mathrm{L}}^{2}} \sim 2.6 \times 10^{-11} \mathrm{erg} \mathrm{cm}^{-2} \mathrm{~s}^{-1}$,

which is much too low with respect to the measured one.

\section{The supernova scenario, part one}

The basis of the supernova scenario is twofold. First, the Milagro excess flux comes from the right direction in the sky: hot spots A and B are about 50 degrees apart, and nicely encompass Geminga (e.g., Bignami \& Caraveo 1996). The pulsar has a nonnegligible proper motion ( $125 \mathrm{~km} \mathrm{~s}^{-1}$ at a distance of 155 parsec) so that its position at birth was different from the present one, the more so if the birthplace was close to the Sun. For the cases of interest, the displacement is around 20-30 degrees towards the south of region A. Such angular distances do not seem implausible in view of the effect of the magnetic field on the arrival direction. Second, a $10 \mathrm{TeV}$ proton diffusing in a $1 \mu \mathrm{G}$ magnetic field in the Bohm regime (i.e., $D \sim c \times r_{\mathrm{L}}$ ) reaches an $e$-folding distance of 65 parsec in the time elapsed since the Geminga supernova explosion $\left(t_{\mathrm{exp}}=3.4 \times 10^{5} \mathrm{yr}\right.$, if the pulsar spin down age is adopted):

$R=\sqrt{4 D t_{\exp }}=\sqrt{4 c r_{\mathrm{L}} t_{\exp }}=65 \mathrm{pc}$.

The diffusion of cosmic rays in the Galaxy (e.g., De Marco et al. 2007) is described with two diffusion coefficients, $D_{\|}$and $D_{\perp}$, parallel and orthogonal to the magnetic field, respectively. The coefficients may be written as

$D_{\|} \sim c \times \lambda, \quad D_{\perp} \sim c \times \frac{r_{\mathrm{L}}^{2}}{\lambda}$

where $\lambda$ is the mean free path along the magnetic field. Usually $\lambda$ is taken much greater than $r_{\mathrm{L}}$, so that $D_{\|}$is much larger than $D_{\perp}$ and the diffusion is strongly anisotropic. However, there are circumstances where the two become equal to each other and the diffusion is isotropic in the Bohm regime: this happens in the limiting case of a very chaotic magnetic field, with $(\delta B / B) \sim 1$ over distances $\sim r_{\mathrm{L}}$, so that $\lambda \sim r_{\mathrm{L}}$. It might be argued that the explosion of the Geminga supernova in the relatively recent past has stirred the local interstellar magnetic field up to the required level of chaos. We pursue this hypothesis as a zeroth order assumption, useful for simple calculations, and a more articulated scenario will be presented in the next section.

The present distance to Geminga is estimated to be $155_{-35}^{+60} \mathrm{pc}$, so we must assume a non negligible velocity of the pulsar in the positive radial direction, equal to at least $160 \mathrm{~km} \mathrm{~s}^{-1}$. On the one hand, such a radial velocity is discordant with the morphology of the Geminga trail (Caraveo et al. 2003), which suggests a velocity vector within 30 degrees from the plane of the sky. Based on this result, Pellizza et al. (2005) put a lower limit of $90 \mathrm{pc}$ on the distance from the Sun at which the supernova explosion might have occurred. On the other hand, a radial velocity as high as assumed here does not seem implausible with respect to the measured transverse velocity; moreover, an even higher value has been suggested (Gehrels \& Chen 1993) in an attempt to relate the Geminga supernova to the formation of the Local Bubble. In any case, the scenario proposed in the next section can accomodate a distance at the lower limit of Pellizza et al. (2005).

The density distribution of particles diffusing with a constant coefficient in a 3-dimensional region is

$n(r, t)=N \frac{2}{3 \sqrt{\pi}} \frac{\mathrm{e}^{-\left(\frac{r^{2}}{4 D t}\right)}}{\frac{4 \pi(4 D t)^{3 / 2}}{3}}$

where $N$ is the total number injected in a small volume at $r=$ $0, t=0$. If $\epsilon$ is the energy of the particles, the net flux at radius $r$ and time $t$ is

$\Phi=-\epsilon D \frac{\partial n(r, t)}{\partial r}=\epsilon n(r, t) \frac{r}{2 t}$.

If we set $t=t_{\exp }, \epsilon=10 \mathrm{TeV}$, and $r=R$ and require agreement with the right hand side of Eq. (1), we deduce $n$ from Eq. (7) and $N$ from Eq. (6). Finally, we assume that the excess cosmic rays have the same spectrum as the background cosmic rays, and obtain the following estimate for the cosmic ray output $E$ of the Geminga supernova

$n\left(R, t_{\text {exp }} ; \epsilon\right)=6.7 \times 10^{-18} \mathrm{~cm}^{-3}, \quad N(\epsilon)=1.7 \times 10^{45}$

$E=1.5 \times 10^{49} \mathrm{erg}$.

This estimate is perfectly in line with the commonly required efficiency (about 1\%) with which a supernova energy output must be channeled into cosmic rays if indeed supernovae are to maintain the Galactic cosmic ray reservoir.

The irregular distribution of the excess flux, and especially the presence of two disjoint hot spots, is perhaps a consequence of large-scale irregularities in the background medium and background magnetic field: the shape of the diffusing cloud must be much more complex than a perfect sphere.

A final comment is in order about assuming a spectrum of the excess cosmic rays similar to the one of the background cosmic rays, while the Milagro data indicate a much flatter slope (1.5 versus 2.6) and a cutoff above several TeV. The qualitative explanation that we propose has to do with the dependence of $r_{\mathrm{L}}$ and $D$ on the particle energy. If at about $10 \mathrm{TeV}$ the $e$-folding point of the diffusing cloud profile has reached the Sun, at much higher (lower) energies the $e$-folding point is much beyond (before) the Sun position. The ratio of the excess flux at a generic energy to the one at the fiducial energy $(10 \mathrm{TeV})$ can be expressed as a function of the ratio of the relevant diffusion coefficients $D$ and $D_{10}$

$\left(\frac{D_{10}}{D}\right)^{3 / 2} \mathrm{e}^{\left(1-\frac{D_{10}}{D}\right)}$

so that in both limits the excess flux is diminished with respect to the fiducial case.

\section{The supernova scenario, part two}

Drury \& Aharonian (2008) have raised two important objections to the scheme presented above. First, a diffusing cloud of cosmic rays would produce a very wide signal in the sky, instead of the relatively narrow hot spots detected by Milagro. Second, while diffusion in the Bohm regime is thought to occur in peculiar regions, it cannot be the general process governing the propagation of cosmic rays across large distances. They suggest that the hot 
spots might be due to excess cosmic rays streaming almost freely from a magnetic nozzle along a diverging field; the source of excess cosmic rays should be relatively nearby (100 pc or less), located at the nozzle or behind it, and its energy content should be a fraction of a supernova output.

We note that the cosmic rays cannot stream freely all the way from the source to the Sun, because the propagation time would be too short, less than a thousand years, and such a young supernova remnant could not remain unnoticed. Moreover, the scenario of Drury \& Aharonian (2008) does not provide an explanation for the peculiar spectral shape of the Milagro signal. One must assume that propagation occurs by diffusion from the source to the magnetic nozzle, over a time long enough to allow the dissipation of the SNR. This initial part of the propagation process is very similar to what we discussed in the previous section. In particular, diffusion would again act as a "passband" filter in energy, producing a hard spectrum with a high energy cutoff, analogous to the observed one. In the new scheme we can relax the Bohm assumption $\lambda \sim r_{\mathrm{L}}, D_{\|} \sim D_{\perp}$, and can accomodate a wider range of values for the distance of the supernova: indeed, at variance with the previous scheme, now the diffusing cloud of cosmic rays does not need to propagate from the supernova to the Sun, but only from the supernova to the magnetic nozzle. More precisely, diffusion is only needed from the rim of the supernova remnant to the magnetic nozzle, and across-field diffusion (which is the slowest process of all) is needed only from the rim of the SNR to the first "useful" magnetic line (see Fig. 1).

We conclude by arguing that it is unlikely that the supernova responsible for the excess cosmic rays was not the same that produced Geminga. In a cone with vertex on the Sun, axis in the direction of Geminga, height 150 , and base radius $50 \mathrm{pc}$, we expect less than 0.01 supernovae in $3.4 \times 10^{5}$ years for a Galactic rate of $0.01 \mathrm{yr}^{-1}$.

In Fig. 1 we schematize the proposed geometry. The upper panel is the projection of the anticenter region on the plane that, being orthogonal to the Galactic plane, contains the present positions of the Sun and Geminga (the latter at the "close" value of $120 \mathrm{pc}$ ). The lower panel is the projection of the same region on the Galactic plane itself. The directions of the Milagro hot spots are indicated, with the exception of hot spot B, which is not drawn in the lower panel since it is very wide in Galactic longitude and would cover all other elements. The two stars mark two possible positions of the supernova explosion, placed at distances of 90 and $65 \mathrm{pc}$ from the present position of the Sun, respectively. The circles have radii of $10 \mathrm{pc}$, and indicate the volume occupied by fully developed supernova remnants. A possible magnetic nozzle is sketched with heavy lines: one sees that the cosmic rays, after leaving the remnant, need only diffuse across the field for very few parsecs before catching the right field line and propagating to the Sun.

Region B is 50 degrees away from Region A and could have a different origin. However, this hypothesis would entail several additional ad hoc assumptions and would, in any case, conflict with the low probability of multiple nearby supernova explosions. It is still more economical to attribute both regions to the same source. Then the large angular separation should be ascribed to large irregularities of the magnetic field in the Solar vicinity: some of the field lines diverging from the "primary" magnetic nozzle intersect the line of sight to region B, as shown in the upper panel, and could fuel a "secondary" magnetic nozzle in that position. Admittedly, this is another epicycle in the model, but it would have to be invoked in any case, even if region $\mathrm{B}$ were fueled by a source independent of region $\mathrm{A}$.

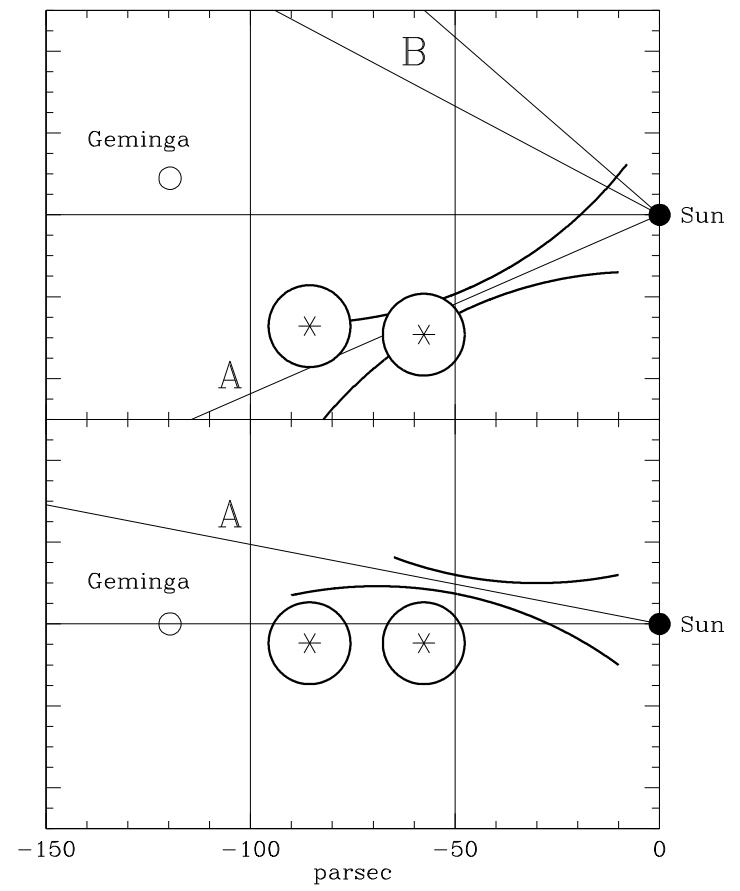

Fig. 1. Projection of the anticenter region on the meridian plane at Galactic longitude $195^{\circ}$ (upper panel) and on the Galactic plane (lower panel). See text for details.

\section{Conclusions}

Our discussion does not prove that the Milagro anticenter hot spots are a transient relic of the explosion of the Geminga supernova. However, it does provide a consistent framework for an experimental result that would otherwise remain unexplained.

If our proposed picture were indeed true, one would conclude that after all supernovae do produce cosmic rays with an efficiency of about $1 \%$. We could see this transient relic only because the supernova that gave birth to the Geminga pulsar exploded very nearby, and not very long ago. Because of such lucky circumstances, the long sought "smoking gun" connecting cosmic rays with supernovae would finally be at hand. Indeed, it could be said that while looking for the "smoking gun" we were hit by the bullets themselves.

Acknowledgements. We would like to thank the friends and colleagues who read our manuscript, almost equally divided between strongly supportive and strongly skeptical. They are Pasquale Blasi, Gabriele Ghisellini, Franco Pacini, Malcolm Walmsley and Lodewijk Woltjer, but we will not disclose who said what.

We also thank the referee, Patrizia Caraveo, for her patience, accuracy, and openmindedness in dealing with our wild ideas.

\section{References}

Abdo, A. A., et al. 2008, PhRvL, submitted [arXiv:0801.3827]

Axford, W. I., \& Suess, S. T. 1994, EOS, 75, 587

Bignami, G. F., \& Caraveo, P. A. 1996, ARA\&A, 34, 331

Caraveo, P. A., Bignami, G. F., DeLuca, A., et al. 2003, Science, 301, 1345

De Marco, D., Blasi, P., \& Stanev, T. 2007, JCAP, submitted [arXiv: 0705.1972]

Drury, L. O'C., \& Aharonian, F. A. 2008, APh, accepted [arXiv:0802 .4403]

Gehrels, N., \& Chen, W. 1993, Nature, 361, 706

Longair, M. S. 1981, High Energy Astrophysics (Cambridge University Press, Cambridge) 128

Ngobeni, M. D., \& Potgieter, M. S. 2008, AdSpR, 41, 373

Pellizza, L. J., Mignani, R. P., Grenier, I. A., \& Mirabel, I. F. 2005, A\&A, 435, 625 